\title{
The role of neoadjuvant chemoradiotherapy vs chemotherapy in cancer of oesophagus
}

\author{
Saini $\mathbf{G}^{1 *}$, Shukla R ${ }^{1}$, VPareek VV' and Goswami $V^{3}$ \\ ${ }^{1}$ Department of Radiation Oncology, Max Institute of Cancer Care, Vaishali and Patparganj, Delhi NCR, India \\ ${ }^{2}$ Department of Radiation Oncology, All India Institute of Medical Sciences, Delhi, India \\ ${ }^{3}$ Department of Medical Oncology, Max Institute of Cancer Care, Vaishali and Patparganj, Delhi NCR, India
}

\begin{abstract}
Oesophageal malignancies are accounted as the eighth most common malignancy across the globe and the sixth leading cause of cancer-related deaths. The mortality to incidence ratio noted to be 0.88 The momentum towards neoadjuvant and adjuvant treatments is therefore understandable. Neoadjuvant radiochemotherapy (nRCT) has emerged as a standard of care in most parts of the world and we review the strength of evidence in favour of the same viz a viz neoadjuvant chemotherapy.
\end{abstract}

\section{Introduction}

Oesophageal malignancies are accounted as the eighth most common malignancy across the globe and the sixth leading cause of cancer-related deaths. The mortality to incidence ratio noted to be 0.88 [1]. Besides the difference in the histological entities, oesophageal malignancies have seen a difference in the incidence across the globe with varied patterns of incidence in the West and the East with squamous cell carcinoma (SCC) being the most common pathology in Asia and adenocarcinoma in North America and Europe. Surgery remains the mainstay of definitive treatment. The anatomical location of oesophagus in close proximity to many critical organs with an absence of serosal lining makes spread by direct extension quite common which in turn makes R0 resection difficult for many patients. The rich submucosal network of lymphatics also makes lymph node metastases quite common. Therefore it is understandable that historically potential resectability has been about 30 to $40 \%$ only with $30-50 \%$ rates of positive margins leading to five-year survival rates of just 15 to $20 \%$ [2-4]. The momentum towards neoadjuvant and adjuvant treatments is therefore understandable. Neoadjuvant radiochemotherapy (nRCT) has emerged as a standard of care in most parts of the world and we review the strength of evidence in favour of the same viz a viz neoadjuvant chemotherapy.

\section{Neoadjuvant radiochemotherapy}

In recent decades, several studies have investigated the benefit of nRCT for oesophageal cancer. Randomised controlled trials comparing nRCT followed by surgery with surgery alone, were initiated in first in 1986 and 1989 [5,6]. After nRCT plus surgery, Apinop et al. reported pathologically complete response (pCR) rate of $27 \%$ and Urba et al. reported it as $28 \%$ not significantly Improving median survival and three-year overall survival between both groups. However, both studies were not powered for detecting small changes in overall survival.

Following this, two sufficiently powered trials were performed $[7,8]$ Walsh et al. randomised 113 patients with oesophageal adenocarcinoma between nRCT ( $40 \mathrm{~Gy}$, administered in 15 fractions over a three-week period with two courses of chemotherapy in weeks 1 and 6 (Cisplatin with fluorouracil) plus surgery versus surgery alone [7]. Over a median follow-up of 10 months. A significant improvement in median overall survival ( 16 vs. 11 months, $\mathrm{p}<0.01)$ and three-year overall survival ( $32 \%$ vs. $6 \%, \mathrm{p}<0.01)$ was reported for patients receiving nRCT. Burmeister et al. [8] reported randomised results of 256 patients with adenocarcinoma or squamous cell ca randomised between nRCT (one cycle of cisplatin and fluorouracil with concurrent 35 Gy in 15 fractions) followed by surgery versus surgery alone. A low pCR rate of $16 \%$ was observed and $80 \% \mathrm{R} 0$ resections were observed in in patients receiving nCRT viz a viz 59\% of patients after surgery alone $(\mathrm{p}<0.001)$. Median overall survival was not significantly different between both groups (22.2 vs. 19.3 months, $\mathrm{p}=0.570$ ). In 2010, Lv et al. [8] reported randomised data on 238 patients with squamous cell carcinoma and reported R0 resection rate of $97.4 \%$ as compared with $80 \%$ in surgery alone group. They reported a significantly better median overall survival (53 vs. 36 months, $\mathrm{p}<0.005)$ and five-year overall survival $(43.5 \%$ vs. $33.8 \%, p^{1 / 4} 0.0402$ ) in favour of the nRCT plus surgery group. Lee et al. reported randomised data of 101 patients with squamous cell carcinoma. Between nRCT (two cycles of cisplatin and fluorouracil with concurrent 45.6 Gy radiotherapy in 38 fractions) followed by surgery versus surgery alone [9]. The R0 resection rate after nRCT compared to primary surgery was $100 \%$ vs. $87.5 \%$ ( $\mathrm{p}=0.037$ ) with $43 \% \mathrm{pCR}$ rates. However, the median overall survival was comparable (28.2 vs. 27.3 months). An important stride in nRCT was taken with reporting of the the Dutch CROSS trial [10,11]. Between 2004 and 2008, 366 patients with locally advanced squamous cell- or adenocarcinoma of the oesophagus or oesophagogastric junction were randomised between

${ }^{\star}$ Correspondence to: Gagan Saini, Department of Radiation Oncology, Max Institute of Cancer Care, Vaishali and Patparganj, Delhi NCR, India, E-mail: drgagansaini@gmail.com

Key words: perioperative, esophagus, toxicity

Received: January 19, 2020; Accepted: January 31, 2020; Published: February 06, 2020 
nRCT (five weekly cycles of carboplatin and paclitaxel and 41.4 Gy radiotherapy in 23 fractions) followed by surgery versus surgery alone. R0 resections were more common in nRCT arm versus surgery alone arm $(92 \%$ vs. $69 \%, \mathrm{p}<0.001)$ with $29 \%$ pCR rates. The median overall survival was better in the nCRT group (48.6 vs. 24 months, $\mathrm{p}<0.003$ ) [11]. The Five-year overall survival in nRCT arm was $47 \%$ compared to $33 \%$ in the surgery alone arm with similar postoperative mortality rates ( $4 \%$ vs. $4 \%, \mathrm{p}=0.700$ ). Unlike the dutch CROSS trial that included patients with locally advanced cancer of esophagus, the French FFCD 9901 trial included 195 early stage (stages I or II) oesophageal cancer patients with squamous cell carcinoma or adenocarcinoma. They were randomised between nRCT (two cycles of cisplatin and fluorouracil with concurrent $45 \mathrm{~Gy}$ radiotherapy in 25 fractions) plus surgery versus surgery alone. R0 resection rates were comparable between both groups (93.8\% vs. $92.1 \%, \mathrm{p}=0.749)$ with $\mathrm{pCR}$ rates of $35.8 \%$ with significantly higher postoperative deaths occurred in the nRCT plus surgery arm ( $11.1 \%$ vs. $3.4 \%, p=0.049)$. A possible explanation for this is the fact that most centers included were not high-volume [12]. No increased mortality rate was reported inthe CROSS study where carboplatin with paclitaxel was used instead of cisplatin with fluorouracil (radiation schedules were comparable). The number of patients proceeding to surgery in FFCD study were just $86 \%$ compared to $92 \%$ in CROSS trial. The median overall survival in the FFCD 9901 trial was not significantly different between the $\mathrm{nRCT}$ plus surgery group and the surgery alone group (31.8 vs. $41.2, \mathrm{p}=0.940)$.

Many of the above studies were not sufficiently powered to show significant advantage of nRCT followed by surgery over surgery alone, resulting in contradictory results. Sjoquist et al. reported their metaanalysis to assess the benefit of nRCT for operable oesophageal cancer patients [13]. Thirteen studies included compared chemoradiotherapy plus surgery versus surgery alone with a total of 1932 patients. Most studies used a neoadjuvant regimen of cisplatin and fluorouracil with concurrent 20-50.4 Gy radiotherapy. They reported a pooled hazard ratio (HR) for all-cause mortality of $0.78(95 \% \mathrm{CI} 0.70-0.88, \mathrm{p}<0.0001)$, which is the equivalent of a two-year overall survival benefit of $8.7 \%$ when patients are treated with nRCT followed by surgery compared to surgery alone. The HR for the overall indirect comparison of all-cause mortality for nRCT versus neoadjuvant chemotherapy (nCT) was 0.88 $(0.76-1.01 ; \mathrm{p}=0.07)$. This technically means that there is no significant difference between two approaches of nRCT or nCT.

A recent meta-analysis by Jing et al. from China reported comparison between nRCT and nCT approaches by including only randomised trials. Three eligible randomized controlled trials were included with a total of 375 patients ( $189 \mathrm{nRCT}, 186 \mathrm{nCT}$ ). The HR for the overall indirect comparison of all-cause mortality for $\mathrm{nRCT}$ versus $\mathrm{nCT}$ was $0.75(0.40-1.41 ; \mathrm{p}=0.113)$. Once again the fact that there is no significant difference between the two approaches of nRCT and nCT brings us to our next section.

\section{Neoadjuvant chemotherapy $v s$ radiochemotherapy}

Oncologists commonly advise peri-operative or neoadjuvant chemotherapy ( $\mathrm{nCT}$ ) basing the recommendation on the MAGIC trial [14]. The MAGIC trial reported results on 503 patients with adenocarcinoma of the distal oesophagus, oesophagogastric junction or stomach. Patients were randomised between perioperative chemotherapy (three preoperative and three postoperative cycles of epirubicin, cisplatin and fluorouracil) plus surgery versus surgery alone. The median survival after a median follow-up period of 48 months was significantly better in the perioperative chemotherapy group than in the surgery alone group (23.5 vs. 19.5 months, $\mathrm{p}<0.009)$. However since $74 \%$ of patients included in the MAGIC trial had adenocarcinoma of the stomach, it is not equitable to extrapolate these results to adenocarcinomas of the distal oesophagus or oesophagogastric junction. In 2002, Medical Research Council Oesophageal Cancer Working Group reported results of a randomised controlled trial [15] on 802 patients with resectable oesophageal squamous cell- or adenocarcinoma. Patients were randomised between nCT (two cycles of cisplatin and fluorouracil) followed by surgery versus surgery alone. After a median follow-up of 37.4 months, median survival was significantly better in the preoperative chemotherapy group than in the surgery alone group ( 16.8 vs. 13.3 months, $\mathrm{p}<0.004)$. R0 resections were achieved in $65 \%$ of patients in the nCT group and in $56 \%$ of patients in the surgery alone group with a pCR rate of $4 \%$ with nCT. Long term follow-up reported in 2009 reports an absolute benefit of 5\% with nCT [16]. The results of RTOG 8911 trial [17] contradict results of OEO2 trial. In the RTOG 8911 trial, 440 patients with oesophageal squamous cell- or adenocarcinoma were randomised between perioperative chemotherapy (three preoperative cycles of cisplatin and fluorouracil and for responders two additional postoperative cycles of cisplatin and fluorouracil) followed by surgery versus surgery alone. After a median follow-up of 46.5 months, median overall survival was comparable between the perioperative chemotherapy group and the surgery alone group (14.9 vs.16.1 months, $\mathrm{p}=0.53$ ). R0 resections were reported in $78 \%$ of patients undergoing surgery in the perioperative chemotherapy group and in $62 \%$ of patients in the surgery alone group with a pCR rate of $2.5 \%$ after a minimum of one cycle chemotherapy. The discrepancy in outcome between the OEO2 and RTOG 8911 trial may be explained by the fact that patients receiving chemotherapy in the RTOG 8911 trial were less likely to undergo surgery since $20 \%$ of patients in the perioperative group did not proceed to surgery in contrast with $4 \%$ in the surgery alone group. This rate was only $10 \%$ and $3 \%$ respectively in the perioperative chemotherapy and surgery alone group as reported in the $\mathrm{OEO} 2$ trial (vs. $3 \%$ in the surgery alone group).

Sjoquist et al. reported meta-analysis of pooled data of trials investigating neoadjuvant chemotherapy [13]. Ten studies with a total of 2062 patients comparing chemotherapy followed by surgery versus surgery alone were included. The MAGIC trial was excluded because there were just $26 \%$ patients with oesophageal or junctional cancer. Also, the operations performed were mostly gastrectomies. This metaanalysis reported a survival benefit for patients treated with preoperative chemotherapy of $5.1 \%$. When comparing both treatment strategies only two studies directly comparing both neoadjuvant therapies were identified $[18,19]$. These studies were both underpowered for overall survival because just 75 and 119 patients were enrolled. Neither study reported a significant benefit for one of both neoadjuvant regimens by direct comparison. Both authors $[18,19]$ concluded nRCT to be a better option than nCT. However with a total of 2220 patients, the pooled hazard ratio for all-cause mortality in the nRCT followed by surgery group was $0.88(0.76-1.01, \mathrm{p}=0.070)$ compared to the $\mathrm{nCT}$ group thus making $\mathrm{nRCT}$ and $\mathrm{nCT}$ statistically comparable neoadjuvant options with a criticism of inclusion of trials that themselves reported superior results with nRCT.

In 2019 Von Döbeln GA et al. [20] reported results of NeoRes I which is a randomized phase II trial comparing nRCT with nCT in the treatment of resectable cancer of the esophagus or gastroesophageal junction. Patients with biopsy-proven adenocarcinoma or squamous cell carcinoma, T1N1 or T2-3N0-1 and M0-M1a were randomized to receive three 3-weekly cycles of cisplatin and fluorouracil with or without the addition of concurrent radiotherapy $40 \mathrm{~Gy}, 2 \mathrm{~Gy} /$ fraction, 
5 days a week, followed by surgery. Primary endpoint was pCR rate in the primary tumor. Survival and recurrence patterns were evaluated as secondary endpoints. A total of 181 patients were enrolled. nRCT arm reported more pCR (28\% vs. $9 \%)$. Five-year progression-free survival was $38.9 \%$ in the nRCT group versus $33.0 \%(\mathrm{P}=0.82)$ in the $\mathrm{nCT}$ group and the five-year overall survival was $42.2 \%$ versus $39.6 \%(\mathrm{P}=0.60)$ respectively. There were no differences in recurrence patterns between the treatment groups.

A systematic review by Jing et al. [21] published in 2019 only analysed three eligible randomized controlled trials that compared nRCT with nCT and included a total of 375 patients. They reported that $\mathrm{nRCT}$ results in higher $\mathrm{pCR}$ rate and higher $\mathrm{R} 0$ resection rates when compared with nCT without significantly affecting survival in resectable esophageal and junctional cancer. While the sample size seems to be small, the included studies are all RCTs, so the level of data and conclusions obtained is very high.

Therefore it is clear that $\mathrm{nRCT}$ produces larger $\mathrm{pCR}$ rates and $\mathrm{R} 0$ resections than $\mathrm{nCT}$ but the same doesn't translate into a more superior survival benefit. The most promising report of the dutch CROSS trial uses paclitaxel and carboplatin as concurrent chemotherapy regimen whereas most other studies use cisplatin and fluorouracil. For nCT the most promising results are from the FLOT study where they used docetaxel along with cisplatin and fluorouracil [22].

The proponent for nRCT allude to the superior pCR and R0 resection rates that are clinically encouraging whereas the proponents for nCT quote the statistical non-superiority of either approach and higher toxicity with nRCT.

It is possible that nRCT may increase treatment-related toxicity and postoperative complication rates. Stahl et al. [18] reported numerically higher in-hospital mortality by adding preoperative radiation therapy (two of 52 patients undergoing surgery [3.8\%] vs five of 49 patients undergoing surgery [10.2\%] in $\mathrm{nCT}$ and $\mathrm{nRCT}$, respectively; $\mathrm{p}=0.26$ ). The NeoRes I trial [20] reported no differences between the treatment arms in terms of frequency of postoperative complications, although complications appeared to be more severe in the nRCT arm. Fan et al. meta-analysis [23] showed an increase in perioperative mortality and postoperative complications when radiotherapy was utilized. Kumagai et al. [24] hypothesized that preoperative nCRT significantly increased postoperative mortality and treatment-related death in patients with ESCC, potentially explaining the lack of survival benefit despite better tumor response. They however concluded that there is no difference in toxicity overall between nRCT Vs nCT approach. Burmeister et al. [19] compared complication rates and showed no statistical difference between the two treatment modes $(\mathrm{p}>0.05)$. More treatment-related toxicity and postoperative complication rates in nRCT might be the reason for that a higher $\mathrm{pCR}$ and $\mathrm{R} 0$ resection rate cannot confer a survival advantage.

Therefore, in search for a scientific recourse to qualifying patients for nRCT in clinical practice perhaps we should strictly follow the patient related eligibility criteria laid down in the landmark trial like the dutch CROSS trial [11] to minimize the morbidity and maximize the benefit for our patients. The patient related criteria for eligible patients were aged 75 years or younger with adequate haematological, renal, hepatic, and pulmonary function; and a WHO performance score of 2 or better, without a past or present history of other malignancy. The main exclusion criteria were past or current history of malignancy other than the oesophageal malignancy, previous chemotherapy and/ or radiotherapy, and weight loss of more than $10 \%$ of the original bodyweight.

Eelke et al. [25] published a comparison of outcomes on patients receiving Dutch CROSS protocol of nRCT outside the trial compared with the patients within the trial who received nRCT. They reported no statistically significant differences in adverse events (pulmonary, cardiac, or anastomotic complications) or survival between the comparison cohorts. Another report by de Heer et. [26] enrolled 161 patients with locally advanced EC (T1N1-3/T2-4aN0-3/M0) treated with the CROSS schedule followed by esophagectomy. There were two groups, group 1 met the CROSS criteria and the group 2 met the extended eligibility criteria, i.e. a tumor length greater than $8 \mathrm{~cm}$, more than $10 \%$ weight loss, more than 2-4 cm extension in the stomach, celiac lymph node metastasis, and/or age over 75 years. The study assessed the differences in nRCT-associated toxicity, 90-day postoperative mortality. The prognostic factors for OS was assessed using Cox multivariate analysis. No difference was found in nRCT-associated toxicity $(\mathrm{P}=0.117)$, postoperative complications ( $\mathrm{P}=0.783)$, and 90 -day mortality $(\mathrm{P}=0.492)$. The OS differed significantly $(\mathrm{P}=0.004)$, with a median of 37.3 months for group 1 and 17.2 months for group 2. However the tumor related factors of pathologic $\mathrm{N}$ stage $(\mathrm{P}=0.023)$, pathologic $\mathrm{T}$ stage $(\mathrm{P}=0.043)$, and group $2(\mathrm{P}=0.008)$ were independent prognostic factors for OS. In another report by Von Döbeln GA et al. on patients of NeoRes1 trial authors reported results of a cardiac stress test on a stationary bicycle and a spirometry that were performed before and after neoadjuvant treatment and 1 to 2 years later after surgery provided that the cancer had not recurred. While they reported impairment in pulmonary function measured as vital capacity and forced expiratory volume in 1 second and a decrease in exercise capacity after neoadjuvant treatment and 1 to 2 years later after surgery. They did not report any differences between $\mathrm{nRCT}$ or $\mathrm{nCT}$ patients.

It is therefore clear that nRCT as per CROSS protocol can be reasonably extrapolated to practice without much perceived increase in morbidity to the patients and that there is no clear evidence of higher post-operative morbidity with nRCT except for the possibility of squamous cell carcinoma.

\section{Future directions}

The controversy on the optimal treatment strategies for operable oesophageal carcinoma patients will remain until well-designed randomised trials are performed which directly compare nRCT according to the CROSS regimen with neoadjuvant chemotherapy according to MAGIC or FLOT. Currently, two ongoing randomised trials are addressing this issue $[27,28]$. The German ESOPEC trial (NCT02509286) aims to randomise 438 patients with locally advanced oesophageal or junctional adenocarcinoma between nRCT plus surgery (CROSS regimen) and perioperative FLOT chemotherapy plus surgery [27]. The Irish NEOAEGIS trial (NCT01726452) aims to randomise 540 patients with locally advanced oesophageal or junctional adenocarcinoma between CROSS and MAGIC or FLOT chemotherapy, followed by surgery [28]. These trials will likely provide evidenc for the optimal neo adjuvant or peri-operative treatment in patients with carcinoma of the oesophagus. The Japanese NExT trial [29] (JCOG 1109, UMIN000009482) aims to randomise 501 patients with mostly locally advanced oesophageal squamous cell carcinoma into three neoadjuvant regimens plus surgery: 1) neoadjuvant chemotherapy with cisplatin and fluorouracil (similar to OEO2 and RTOG 8911), 2) neoadjuvant chemotherapy with fluorouracil, cisplatin and docetaxel (similar to FLOT), and 3) nCRT with cisplatin, fluorouracil and $41.4 \mathrm{~Gy}$ radiotherapy in 23 fractions, 5 fractions a week similar to FFCD 9901). 


\section{References}

1. Bray F (2018) Global cancer statistics 2018: GLOBOCAN estimates of incidence and mortality worldwide for 36 cancers in 185 countries. CA: A Cancer Journal for Clinicians 68: 394-424. [Crossref]

2. Bosset JF, Gignoux M, Triboulet JP, Tiret E, Mantion G, et al. (1997) Chemoradiotherapy followed by surgery compared with surgery alone in squamous-cell cancer of the esophagus. N Engl J Med 17: 161-167. [Crossref]

3. Mariette C, Finzi L, Fabre S, Balon JM, Van Seuningen I, et al. (2003) Factors predictive of complete resection of operable esophageal cancer: a prospective study. Ann Thorac Surg 75: 1720-1726. [Crossref]

4. Hulscher JB, van Sandick JW, de Boer AG, Wijnhoven BP, Tijssen JG, et al. (2002) Extended transthoracic resection compared with limited transhiatal resection for adenocarcinoma of the esophagus. $N$ Engl J Med 21: 1662-1669. [Crossref]

5. Apinop C, Puttisak P, Preecha N (1994) A prospective study of combined therapy in esophageal cancer. Hepatogastroenterology 41: 391-393. [Crossref]

6. Urba SG, Orringer MB, Turrisi A, Iannettoni M, Forastiere A, et al. (2001) Randomized trial of preoperative chemoradiation versus surgery alone in patients with locoregional esophageal carcinoma. J Clin Oncol 19: 305-313. [Crossref]

7. Walsh TN, Noonan N, Hollywood D, Kelly A, Keeling N, et al. (1996) A comparison of multimodal therapy and surgery for esophageal adenocarcinoma. $N$ Engl J Med 15: 462-467. [Crossref]

8. Lv J, Cao XF, Zhu B, Ji L, Tao L, et al. (2010) Long-term efficacy of perioperative chemoradiotherapy on esophageal squamous cell carcinoma. World J Gastroenterol 16: 1649-1654. [Crossref]

9. Lee JL, Park SI, Kim SB, Jung HY, Lee GH, et al. (2004) A single institutional phase III trial of preoperative chemotherapy with hyperfractionation radiotherapy plus surgery versus surgery alone for resectable esophageal squamous cell carcinoma. Ann Oncol 15: 947-954. [Crossref]

10. Van Hagen P, Hulshof MC, van Lanschot JJ, Steyerberg EW, van Berge, et al. (2012) Preoperative chemoradiotherapy for esophageal or junctional cancer. $N$ Engl J Med 366: 2074-2084. [Crossref]

11. Shapiro J, van Lanschot JJB, Hulshof M, van Hagen P, van Berge, et al. (2015) Neoadjuvant chemoradiotherapy plus surgery versus surgery alone for oesophageal or junctional cancer (CROSS): long-term results of a randomised controlled trial. Lancet Oncol 16: 1090-1098. [Crossref]

12. Mariette C, Dahan L, Mornex F, Maillard E, Thomas PA, et al. (2014) Surgery alone versus chemoradiotherapy followed by surgery for stage I and II esophageal cancer: final analysis of randomized controlled phase III trial FFCD 9901. J Clin Oncol 32: 2416-2422. [Crossref]

13. Sjoquist KM, Burmeister BH, Smithers BM, Zalcberg JR, Simes RJ, et al. (2011) Survival after neoadjuvant chemotherapy or chemoradiotherapy for resectable oesophageal carcinoma: an updated meta-analysis. Lancet Oncol 12: 681-692. [Crossref]

14. Cunningham D, Allum WH, Stenning SP, Thompson JN, Van de Velde CJ, et al. (2006) MAGIC Trial Participants. Perioperative chemotherapy versus surgery alone for resectable gastroesophageal cancer. $N$ Engl J Med 6: 11-20. [Crossref]

15. Medical Research Council Oesophageal Cancer Working Group (2002) Surgica resection with or without preoperative chemotherapy in oesophageal cancer: a randomized controlled trial. Lancet 18: 1727-1733. [Crossref]
16. Allum WH, Stenning SP, Bancewicz J, Clark PI, Langley RE (2009) Long-term results of a randomized trial of surgery with or without preoperative chemotherapy in esophageal cancer. J Clin Oncol 30: 5062-5067. [Crossref]

17. Kelsen DP, Ginsberg R, Pajak TF, Sheahan DG, Gunderson L, et al. (1998) Chemotherapy followed by surgery compared with surgery alone for localized esophageal cancer. $N$ Engl J Med 339: 1979-1984. [Crossref]

18. Stahl M, Walz MK, Stuschke M, Lehmann N, Meyer HJ, et al. (2009) Phase II comparison of preoperative chemotherapy compared with chemoradiotherapy in patients with locally advanced adenocarcinoma of the esophagogastric junction. $J$ Clin Oncol 27: 851-856. [Crossref]

19. Burmeister BH, Thomas JM, Burmeister EA, Walpole ET, Harvey JA, et al. (2011) Is concurrent radiation therapy required in patients receiving preoperative chemotherapy for adenocarcinoma of the oesophagus? A randomised phase II trial. Eur J Canc 47: 354-360. [Crossref]

20. von Döbeln GA, Klevebro F, Jacobsen AB, Johannessen HO, Nielsen NH, et al. (2019) Neoadjuvant chemotherapy versus neoadjuvant chemoradiotherapy for cancer of the esophagus or gastroesophageal junction: long-term results of a randomized clinical trial. Dis Esophagus 32. [Crossref]

21. Jing SW, Qin JJ, Liu Q, Zhai C, Wu YJ, et al. (2019) Comparison of neoadjuvan chemoradiotherapy and neoadjuvant chemotherapy for esophageal cancer: a metaanalysis. Future Oncol 15: 2413-2422. [Crossref]

22. Al-Batran SE, Homann N, Pauligk C, Goetze TO, Meiler J, et al. (2019) Perioperative chemotherapy with fluorouracil plus leucovorin, oxaliplatin, and docetaxel versus fluorouracil or capecitabine plus cisplatin and epirubicin for locally advanced, resectable gastric or gastro-oesophageal junction adenocarcinoma (FLOT4): a randomised, phase 2/3 trial. Lancet 11: 1948-1957. [Crossref]

23. Fan M, Lin Y, Pan J (2016) Survival after neoadjuvant chemotherapy versus neoadjuvant chemoradiotherapy for resectable esophageal carcinoma: a meta-analysis. Thorac Cancer 7: 173-181.

24. Kumagai K, Rouvelas I, Tsai JA (2014) Meta-analysis of postoperative morbidity and perioperative mortality in patients receiving neoadjuvant chemotherapy or chemoradiotherapy for resectable oesophageal and gastro-oesophageal junctional cancers. Br J Surg 101: 321-338. [Crossref]

25. Toxopeus E (2018) Outcome of patients treated within and outside a randomized clinical trial on neoadjuvant chemoradiotherapy plus surgery for esophageal Cancer: Extrapolation of a Randomized Clinical Trial (CROSS). Annals of Surgical Oncology 25: 2441-2448. [Crossref]

26. de Heer EC (2017) Effect of Extending the Original Eligibility Criteria for the CROSS Neoadjuvant Chemoradiotherapy on Toxicity and Survival in Esophageal Cancer. Annals of Surgical Oncology 24: 7. [Crossref]

27. Hoeppner J, Lordick F, Brunner T, Glatz T, Bronsert P, et al. (2016) ESOPEC: prospective randomized controlled multicenter phase III trial comparing perioperative chemotherapy (FLOT protocol) to neoadjuvant chemoradiation (CROSS protocol) in patients with adenocarcinoma of the esophagus (NCT02509286). BMC Canc 16: 503. [Crossref]

28. Reynolds JV, Preston SR, O'Neill B, Baeksgaard L, Griffin SM, et al. (2017) ICORG 10-14: NEOadjuvant trial in adenocarcinoma of the esophagus and oesophago gastric junction international study (Neo-AEGIS). BMC Canc 17: 401

29. Nakamura K, Kato K, Igaki H, Ito Y, Mizusawa J, et al. (2013) Three-arm phase III trial comparing cisplatin plus 5-FU (CF) versus docetaxel, cisplatin plus 5-FU (DCF) versus radiotherapy with $\mathrm{CF}$ (CF-RT) as preoperative therapy for locally advanced esophageal cancer (JCOG1109, NExT study). Jpn J Clin Oncol 43: 752-755. [Crossref]

Copyright: $(2020$ Saini G. This is an open-access article distributed under the terms of the Creative Commons Attribution License, which permits unrestricted use, distribution, and reproduction in any medium, provided the original author and source are credited. 